\title{
Study on the Condensation of Different Hydroxy Aromatic Aldehydes with 2-Substituted 2-Oxazolin-5-ones Generated in situ
}

\author{
Pubanita Bhuyan, Pradeep K. Tripathy* \\ Department of Chemistry, North Eastern Regional Institute of Science and Technology, Nirjuli, Itanagar, India \\ Email address: \\ Pkt_chem@yahoo.co.in (P. K. Tripathy) \\ ${ }^{*}$ Corresponding author
}

To cite this article:

Pubanita Bhuyan, Pradeep K. Tripathy. Study on the Condensation of Different Hydroxy Aromatic Aldehydes with 2-Substituted 2-Oxazolin5-ones Generated in situ. American Journal of Heterocyclic Chemistry. Vol. 5, No. 1, 2019, pp. 1-3. doi: 10.11648/j.ajhc.20190501.11

Received: January 25, 2019; Accepted: March 14, 2019; Published: April 8, 2019

\begin{abstract}
In view to synthesize some bioactive 2-Substituted 4-(hydroxybenzylidene)-2-oxazolin-5-ones through a disciplined route, a study on the condensation of 2-, 3- and 4- hydroxy aromatic aldehydes (6) with 2-Substituted 2-oxazolin-5ones (5) was carried out. 2-Substituted 2-oxazolin-5-ones (5) which are also known as saturated azlactones and unstable, were generated in situ from $\alpha$-N-Acylglycines (1) using various cyclising agents namely ethyl chloroformate (2), benzene sulphonyl chloride (3) and $p$-toluene sulphonyl chloride (4) in dry benzene in presence of triethylamine base. The hydroxyl group at 3and 4- positions of aromatic aldehydes namely 4-hydroxy-3-methoxybenzaldehyde (6a), $m$-hydroxybenzaldehyde (6b)and $p$ hydroxybenzaldehyde (6c) produce 2 -substituted 4-( $p$-hydroxy- $m$-methoxybenzylidene)-2-oxazolin-5-one (8a), 2-substituted-4 ( $m$-hydroxybenzylidene)-2-oxazolin-5-one (8b) and2-substituted-4 ( $p$-hydroxybenzylidene)-2-oxazolin-5-one (8c) respectively as their $(Z)$-isomers, whereas 2-hydroxy aromatic aldehyde namely salicylaldehydeproduces 3-N-acylaminocoumarins (9) on condensation with 2-Substituted 2-oxazolin-5-ones (5) in appreciable yields and good purity. The reaction seems to be initiated by the formation of an adduct $(E)$-2-substituted 4-(o-hydroxybenzylidene-2-oxazolin-5-ones (7), followed by intramolecular 1,5- bond cleavage of the 2-oxazolin-5-one ring by the vicinal phenolic group and subsequent recyclization led to the formation of resultant 3-N-acylaminocoumarins (9). It is noteworthy that free hydroxyl group bearing benzylidene moiety at 4position of 2-oxazolin-5-ones (8) were obtained. All the steps can be carried out in one flask.
\end{abstract}

Keywords: 4-(Hydroxybenzylidene) Azlactones, 3-N-Acylaminocoumarins, Cyclisingagents, Synthons, in situ

\section{Introduction}

In connection with the synthesis of 2-substituted 4(hydroxybenzylidene)-2-oxazolin-5-ones; for example 8 , the chemistry of 3-N-acylaminocoumarins (9) was investigated. 2-Oxazolin-5-ones also called 5(4H)-Oxazolones continue to attract the attention of chemists because of their usefulness as synthons and their different kinds of pharmacological and biological activities [1]. Recently the anticancer activities of some 4-(hydroxybenzylidene)-2-oxazolin-5-ones were also reported [2].

Acetic anhydride-mediated condensation of hippuric acid (1b) with salicyldehyde (6d) is known [3, 12-14] to give a mixture of products 3- Benzoylaminocoumarin (9b) and 4-(oAcetoxybenzylidene)-2-phenyl-2-oxazolin-5-one from which $9 \mathrm{~b}$ can be separated.

However, this reaction was unsuccessful with aceturic acid (1a). The condensation of hippuric acid (1b) with 3- or 4hydroxybenzaldehydes ( $6 \mathrm{~b}$ or $6 \mathrm{c}$ ) in the presence of acetic anhydride and fused sodium acetate afforded 4(acetoxybenzylidene)-2-phenyl-2-oxazolin-5-one as a major product where $-\mathrm{OH}$ group remained blocked by acetyl group $[3,12]$. In order to get free $-\mathrm{OH}$ group in benzylidene moiety at 4-position of the unsaturated azlactone8, a facile and convenient route was developed (Figure 1). 

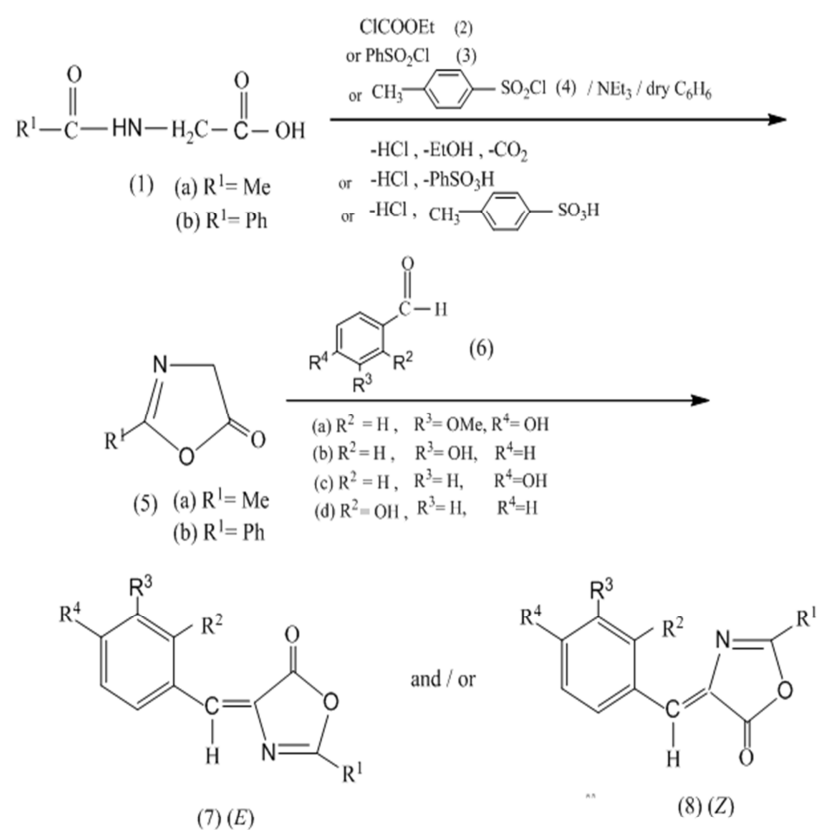

$\mathrm{R}^{2}=\mathrm{OH} \quad \mathrm{R}^{3}=\mathrm{H}, \quad \mathrm{R}^{4}=\mathrm{H}$

(a) $\mathrm{R}^{2}=\mathrm{H}, \mathrm{R}^{3}=\mathrm{OMc}, \mathrm{R}^{4}=\mathrm{OH}$ (b) $\mathrm{R}^{2}=\mathrm{H}, \quad \mathrm{R}^{3}=\mathrm{OH}, \quad \mathrm{R}^{4}=\mathrm{H}$

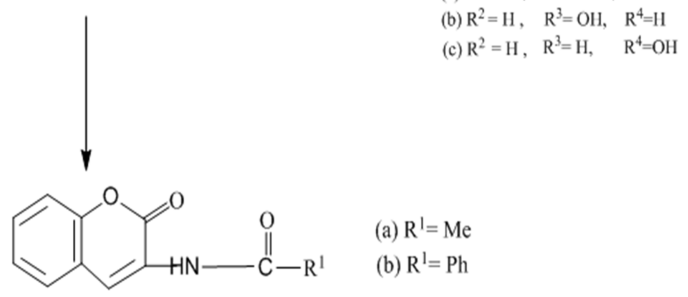

(9)

Figure 1. Syntheses of 2-Subtituted 4-(hydroxybenzylidene)-2-oxazolin-5ones and 3-N acylaminocoumarins.

\section{Method}

All the prepared compounds are known in Literature. The purity of the compounds was verified by TLC (silica gel based) and their melting points. Melting points were recorded by metal block melting point apparatus and are uncorrected. The IR spectra of the compounds were recorded on IR Affinity-1, Shimadzu.

\subsection{Synthesis of (Z)-2-Substituted 4-(hydroxybenzylidene)- 2-oxazolin-5-ones $(8)$ :}

To a suspension of hippuric acid $(1 \mathrm{~b}, 1.79 \mathrm{~g}, 0.01 \mathrm{~mol})$ in dry benzene $(50 \mathrm{~mL})$ containing triethylamine $(3.5 \mathrm{~mL}, 0.025$ mol), $p$-toluenesulphonylchloride $(4,1.9 \mathrm{~mL}, 0.01 \mathrm{~mol})$ was added and the mixture was shaken at room temperature until the hippuric acid crystals disappeared and triethylamine salts separated out which were filtered and washed with benzene $(5 \mathrm{~mL})$. To the benzene filtrate, different aromatic hydroxybenzaldehydes (except 2-Hydroxybenzaldehyde) like vanillin $(6 \mathrm{a}, 1.58 \mathrm{~g}, 0.01 \mathrm{~mol})$ or 3 - hydroxybenzaldehyde (6b, $1.22 \mathrm{~mL}, 0.01 \mathrm{~mol})$ or 4-hydroxybenzaldehyde (6c, 1.22 $\mathrm{mL}, 0.01 \mathrm{~mol}$ ) was added. The mixture was refluxed for 10 minutes. The solution was concentrated to dryness under vacuum. The pasty residue was triturated with chilled $95 \%$ ethanol to afford the title compounds, which was filtered under suction and recrystallized from ethanol.

\subsection{Synthesis of 3-N-Acylaminocoumarins (9)}

To a suspension ofaceturic acid (1a, $1.17 \mathrm{~g}, 0.01 \mathrm{~mol})$ /hippuric acid (1b, $1.79 \mathrm{~g}, 0.01 \mathrm{~mol})$ in dry benzene $(50 \mathrm{~mL})$ containing triethylamine $(3.5 \mathrm{~mL}, 0.025 \mathrm{~mol}$ incase of 3 and 4 ; or $1.82 \mathrm{~mL}, 0.013 \mathrm{~mol}$ in case of 2$)$, ethyl chloroformate (2, $1.05 \mathrm{~mL}, 0.011 \mathrm{~mol})$ or benzenesulphonylchloride $(3,1.77$ $\mathrm{mL}, 0.01 \mathrm{~mol})$ or $p$-toluenesulphonylchloride $(4,1.9 \mathrm{~mL}$, $0.01 \mathrm{~mol}$ )as cyclising agent was added and the mixture was shaken at room temperature until the hippuric acid /aceturic acid crystals disappeared and triethylamine salts separated out which were filtered under suction and washed with benzene $(5 \mathrm{~mL})$. To the benzene filtrate, 2Hydroxybenzaldehyde i.e. salicylaldehyde (6d, $1.22 \mathrm{~mL}, 0.01$ mol) was added. The mixture was refluxed for 2 hours. The solution was concentrated to dryness under vacuum. The pasty residue was triturated with chilled aq. ethanol to afford 3-N-Acetylaminocoumarin (9a) and with chilled $95 \%$ ethanol to afford 3-N-Benzoylaminocoumarin (9b) which were filtered under suction and recrystallized from ethanol.

Yields of pure products are calculated based on the amount of $\alpha-\mathrm{N}$-acylglycines used.

8a: Yield, 25\% (cyclising agent: 4), m.p. $142-144^{\circ} \mathrm{C}$ (Reported [2]: $\left.145-146^{\circ} \mathrm{C}\right), \operatorname{IR}(\mathrm{KBr}): 3606$ (-OH), 1802 (azlactone), $1653(\mathrm{C}=\mathrm{C}) \mathrm{cm}^{-1}$.

8b: Yield, $47 \%$ (cyclising agent: 4), m.p. $144-145^{\circ} \mathrm{C}$ (Reported [2]: $\left.144-145^{\circ} \mathrm{C}\right), \operatorname{IR}(\mathrm{KBr}): 3606$ (-OH), 1802 (azlactone), $1653(\mathrm{C}=\mathrm{C}) \mathrm{cm}^{-1}$.

8c: Yield, 45\% (cyclising agent: 4), m.p. $142-144^{\circ} \mathrm{C}$ (Reported [2]: $\left.144-145^{\circ} \mathrm{C}\right), \quad \mathrm{IR}(\mathrm{KBr}): 3606(-\mathrm{OH}), 1802$ (azlactone), $1653(\mathrm{C}=\mathrm{C}) \mathrm{cm}^{-1}$.

9a: Yield, 12\% (cyclising agent: 2), Yield, 32\% (cyclising agent: 3), Yield, 56\% (cyclising agent: 4), m.p. $202-204^{\circ} \mathrm{C}$ (Reported [9]: $\left.205-206^{\circ} \mathrm{C}\right), \mathrm{IR}(\mathrm{KBr}): 3320(-\mathrm{NH}), 1712$ (CO, coumarin), 1670 (CO, amide), $1628(\mathrm{C}=\mathrm{C}) \mathrm{cm}^{-1}$.

9b: Yield, 35\% (cyclising agent: 2), Yield, 38\% (cyclising agent: 3), Yield, $60 \%$ (cyclising agent: 4), m.p. $172-174^{\circ} \mathrm{C}$ (Reported [9, 13, 14]: $\left.174-175^{\circ} \mathrm{C}\right), \operatorname{IR}(\mathrm{KBr}): 3362(-\mathrm{NH})$, 1710 (CO, coumarin), 1663 (CO, amide), $1605(\mathrm{C}=\mathrm{C}) \mathrm{cm}^{-1}$.

\section{Results}

With a view to converting the unstable 2-substituted 2oxazolin-5-ones (5) obtained by either ethyl chloroformate (2) or benzenesulphonylchloride (3) or $p$ toluenesulphonylchloride (4) mediated cyclisation of $\alpha-\mathrm{N}$ acylamino acids (1), into the more stable 2- substituted 4(hydroxyaryl)methylene-2-oxazolin-5-ones (8), a suitable hydroxy aromatic aldehydes, were added to the reaction mixture which were heated under reflux for about 10 minutes. On work-up, 8obained as pure $(Z)$-isomer in appreciable yields but no targeted product of unsaturated azlactone was obtained on condensation of 5 with 2-hydroxy -benzaldehyde (6d) as reported in literature [4, 5], whereas the reaction afforded 3- Acylamino- coumarins (9) on extension of refluxing the reaction mixture for about 2.0 
hours [6].

\section{Discussion}

It has been found that the formation of 3acylaminocoumarins (9) and 2-substituted-4hydroxybenzylidene-2-oxazolin-5-ones (8) depend on the type of hydroxy aromatic aldehydes (6), cyclizing agents and the reaction conditions. For example, 2-phenyl-2-oxazolin-5one (5b) and 2- hydroxybenzaldehyde (6d) afforded 3-Nbenzoylaminocoumarin (9b) exclusively, irrespective of the cyclocondensing agent used for the generation of 5 . But the formation of 3-N-acetylaminocoumarin (9a) in preparative yield was possible only when aceturic acid (1a) was cyclised with either benzenesulphonylchloride or $p$ toluenesulphonylchloride. The condensation of 2-substituted2-oxazolin-5-ones (5) with 4-hydroxy-3methoxybenzaldehyde (6a) or3-hydroxybenzaldehyde (6b) or 4-hydroxybenzaldehyde (6c) afforded (Z)-isomer of 2substituted 4-(hydroxybenzylidene)-2-oxazolin-5-ones (8) whereas 2-hydroxybenzaldehyde (6d)afforded 3-Nacylaminocoumarins (9) under similar conditions. Further the reaction time varies from 10 minutes to 2 hours to get 8 and 9 respectively.

From the results obtained, it is apparent that 2-substituted 4-(hydroxybenzylidene)-2-oxazolin-5-ones are intermediates in the formation of coumarins (9). Though the synthesis of coumarin (9) through the $(E)$-azlactone (7) is possible, this pathway does not seem to predominate, since the $(Z)$ azlactone (8), which should have also been formed from the thermolabile $(E)$-isomer 7, was not discernible even as a minor product. Because of the stereochemical compulsion, (Z)-isomer 8 would not undergo ring expansion to 9 , unless it is isomerisedphotochemically or by some reagent, thereby ruling out its involvement in the present case. The reaction seems to be initiated by the formation of an adduct, followed by intramolecular 1,5- bond cleavage of the 2-oxazolin-5-one ring by the vicinal phenolic group and subsequent recyclization led to the formation of resultant coumarins (9).

The coumarins obtained have been characterized on the basis of their relavant spectral data. The characteristic IR bands of2- substituted 4- hydroxyarylidene-2-oxazolin-5ones (8) appear at the range of $1790-1810 \mathrm{~cm}^{-1}$, whereas $1710 \mathrm{~cm}^{-1}(\mathrm{CO}$, coumarin)for coumarins (9) are obtained .

The present method for the synthesis of $3-\mathrm{N}-$ acylaminocoumarins (9) is quite convenient since all the steps can be carried out in the same flask unlike some of the known methods [10-12, 15]. The present procedure overcomes some of the disadvantages of the earlier methods regarding speed of the reaction and stereochemical purity of the products. For example, the Erlenmeyer azlactone synthesis employs acetic anhydride for cyclization and it affords a mixture of $(E)$ - and $(Z)$ - isomers of the unsaturated azlactones [7]. Further, acetylation of the free -OH group of aldehydes usually occurs simultaneously which leads to the formation of acetoxybenzylidene moiety at 4- position of $8[3]$. It should be emphasized that the present procedure is simple and straight forward.

\section{Conclusion}

2-Substituted 2-oxazolin-5-ones (5) remain very important starting materials for the construction of various heterocycles and therefore can be used as synthons. A disciplined route for the fast and facile one flask syntheses of 2-substituted-4(hydroxybenzylidene)-2-oxazolin-5-ones (8) and 3acylaminocoumarins (9) were developed with shorter reaction time from 10 minutes to 2 hours and chemical purity and steric integrity of the products were maintained simultaneously. In view of the ready availability of the reactants, mild experimental conditions and good overall yields, the present proposed route appears to be potentially important for the synthesis of 3-N- acylaminocoumarins (9) and hydroxybenzylidene moiety containing azlactones (8).

\section{References}

[1] Sharma N, Banerjee J, Shrestha N and Choudhury D, A review on oxazolone, European J. Biomedical and Pharmaceutical Sciences 2015; 2: 964-87.

[2] Jat RL, Mishra R and Pathak D, International J. Pharmacy and Pharmaceutical Sciences 2012; 4: 378-80.

[3] Erlenmeyer E and Stadlin W, Liebigs. Ann. chem. 1904; 337: 283-85.

[4] Baltazzi E and Devis EA, Chem. Ind. (London) 1962; 929.

[5] Takagaki H, Tanabe S, Asoka $M$ and Takel H, Chem. Lett. 1979; 347-50.

[6] Tripathy PK, Indian J. Heterocyclic Chem.1992; 2: 77-80.

[7] Review: Rao YS and Filler R, Synthesis 1975; 749-64.

[8] Erlenmeyer E and Matter O, Liebigs. Ann. Chem. 1904; 337:271-3.

[9] Tripathy PK and Mukerjee AK, Indian J. Chem. 1987; 26B: 61-62.

[10] Ashare Ram and Mukerjee AK, Indian J. Chem. 1986; 25B: 423-24.

[11] Tripathy PK and Mukerjee AK, Heterocycles 1987; 26:151720.

[12] Mogilaiah K, Prashanthi M and Reddy CH, Indian J. Chem. 2003; 42B: 2126-28.

[13] Marrian DH, Russel PB and Todd AR, Biochem. J. 1949; 45: 533.

[14] Kirby GW, Michael J and Narayanaswami S, J. Chem. Soc. Perkin Trans- 1 1972; 203.

[15] Mukerjee AK and Ashare Ram, Chemical Reviews 1991; 91: $1-24$. 